\title{
Metastatic Secondary Anaplastic Meningioma
}

\author{
Luigina Santorsa' ${ }^{1}$, Filomenamila Lorusso² ${ }^{2}$, Rosa Divella ${ }^{3}$, Antonia Gentile ${ }^{4}$, \\ Antonio Mazzocca ${ }^{3}$, Pasquale Di Fazio5, Laura Troiani'1, Claudio Lotesoriere', \\ Giovanna Campanella1, Junia Bagnoli5, Ivan Lolli*
}

\author{
${ }^{1}$ Medical Oncology, IRCCS “Saverio de Bellis” Castellana Grotte, Bari, Italy \\ ${ }^{2}$ Unit of Radiology, IRCCS “Saverio de Bellis” Castellana Grotte, Bari, Italy \\ ${ }^{3}$ Experimental Oncology, IRCCS “Istituto Tumori Giovanni Paolo II”, Bari, Italy \\ ${ }^{4}$ Department of Emergency and Organ Transplantation, Anatomic Pathology, University of Bari, Bari, Italy \\ ${ }^{5}$ Nuclear Medicine, “Mons. Dimiccoli” Hospital, Barletta, Italy \\ Email: *ivanlolli1@tin.it
}

How to cite this paper: Santorsa, L., Lorusso, F., Divella, R., Gentile, A., Mazzocca, A., Di Fazio, P., Troiani, L., Lotesoriere, C., Campanella, G., Bagnoli, J. and Lolli, I. (2016) Metastatic Secondary Anaplastic Meningioma. Open Access Library Journal, 3: e3170. http://dx.doi.org/10.4236/oalib.1103170

Received: October 24, 2016

Accepted: November 14, 2016

Published: November 17, 2016

Copyright $\odot 2016$ by authors and Open Access Library Inc.

This work is licensed under the Creative Commons Attribution International License (CC BY 4.0).

http://creativecommons.org/licenses/by/4.0/ (c) (7) Open Access

\begin{abstract}
Usually, meningiomas are benign neoplasms and their malignant behavior is limited to local recurrence and brain invasion. The rate of local recurrence increases according to histological grade as reported by WHO criteria while extracranial metastases are rare. Anaplastic meningiomas (AM) represent the most rare and aggressive subtype of all meningiomas. This tumor may progress from lower grade I or II meningiomas (secondary AM) or develops as de novo disease (primary AM). We report a case of extracranial metastatic progression of a heavily treated intracranial secondary anaplastic meningioma. The metastatic progression was detected as unexpected and unsuspected diagnosis because it occurred in absence of local intracranial recurrence. There are no criteria to evaluate the risk for the metastatic spread. In hindsight, a malignant progression in tumor histological grade and a significant increase of ki-67 index represent useful predictors of the aggressive behavior.
\end{abstract}

\section{Subject Areas}

Neurology, Oncology

\section{Keywords}

Anaplastic Meningioma, Chemotherapy, Target Therapy, Stereotactic Radiosurgery

\section{Introduction}

Meningiomas arise from arachnoidal cells of the leptomeninges and account for 13\% $26 \%$ of all primary brain tumors in adults [1]. Although most meningiomas are considered to be slow-growing lesions, they present histologically distinct subsets associated 
with different risks of local recurrence, even after macroscopically complete excision. According to the World Health Organization (WHO) criteria, there are three grades of histologic subtypes of meningiomas with different aggressiveness. About $90 \%$ of all meningiomas are grade I and show pleomorphic features and occasional mitotic figures. This category, usually defined as benign, presents a local recurrence rate between $7 \%$ and $20 \%$. The rest is classified as atypical (grade II) or anaplastic (grade III) tumors. Atypical meningiomas $(4.7 \%-7.2 \%)$ present histological aspect of malignancy with an increased mitotic ratio defined as $\geq 4$ mitoses/10 high power fields (HPF). These tumors display a more aggressive clinical behaviour and have a recurrence rate ranging between $30 \%$ and $40 \%$. Anaplastic meningiomas (AM-WHO-III ${ }^{\circ}$ ) represent the rarest (1\% - 3\% of cases) and the most aggressive subtype with poor prognosis and recurrence rates as high as $50 \%-78 \%$. These tumors show frank malignant features with a high mitotic index ( $\geq 20$ mitoses/10 HPF). They may progress from low-grade meningiomas (secondary AM-WHO-III ${ }^{\circ}$ ) or develop as de novo disease, primary AM-WHO-III ${ }^{\circ}$ [2]. If we consider all grades meningiomas, the incidence of metastases is very low and the risk of metastatic spread increases along with higher grades. An intracranial meningioma usually recurs locally several times before it metastasizes. Here, we report a rare case of a heavily treated intracranial secondary AM-WHO-III ${ }^{\circ}$ with subsequent massive extracranial metastases. The reported case is unusual because the patient's meningioma showed an aggressive behavior after first surgery and adjuvant radiotherapy with a documented rapid malignant progression in histological grade from an atypical to an aggressive form with a poorly differentiated phenotype. Initially it was classified as atypical while the recurrent tumor resulted anaplastic at second surgery. Moreover, the secondary AM-WHO-III ${ }^{\circ}$ spread widely and metastasized pleura, liver and lymph nodes in absence of intracranial progression.

\section{Case Report}

A 56-year-old man was admitted to hospital for clinical evaluation for headache and mild anomic aphasia. The patient's medical history was unremarkable. Magnetic resonance imaging (MRI) was carried out and showed a large solid enhancing extra axial tumor with significant mass effect localized in the left frontal lobe (Figure 1(A)). A macroscopically total surgical resection of tumor was performed. Histopathologic examination revealed a monotonous proliferation of spindle cells with high nucleus/cytoplasmic ratio and prominent nucleoli, displaying a mitotic activity with about 6 mitosis/10HPF and rare foci of necrosis. Immunoistochemistry showed epithelial membrane antigen (EMA) positivity, absence of cytokeratine immunoreactivity and proliferation index (Ki67: 10\%) consistent with diagnosis of atypical meningioma. The patient was referred to adjuvant radiotherapy for a $60 \mathrm{~Gy}$ total dose over 30 fractions. Three months after radiotherapy treatment, the patient presented slight aphasia, moderate right hemiparesis and dysgraphia. A new radiological examination revealed recurrence on the previous surgical site (Figure $1(B)$ ). Therefore, a second surgery with total resection of the tumor was done. Histopathologic examination revealed massive proli- 

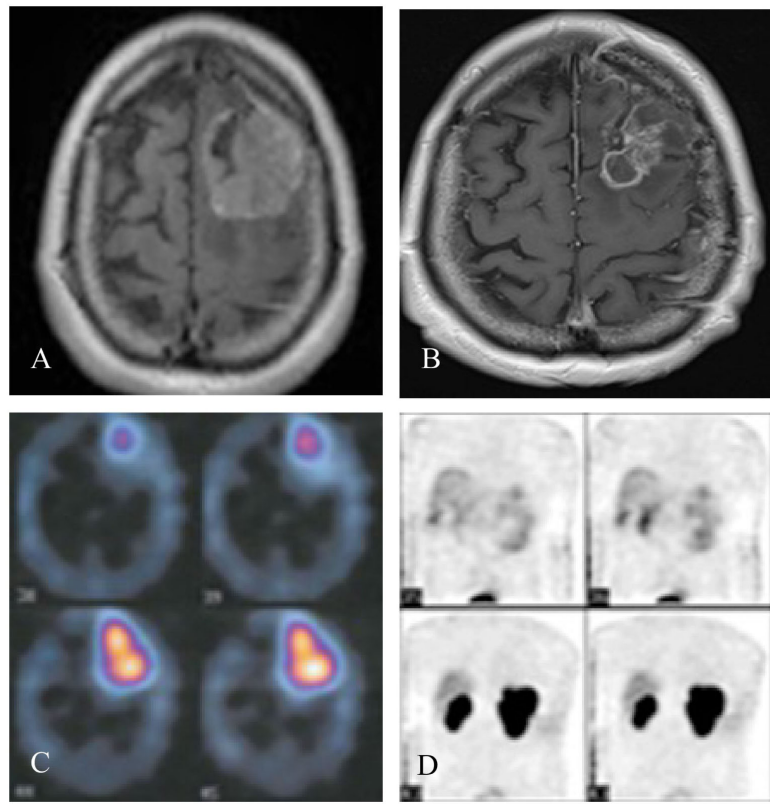

Figure 1. Magnetic resonance imaging (MRI) and 111 Inpenteotride octreoscan (A); enhancing extra-axial tumor in left frontal lobe (baseline); (B) disease recurrence at surgical site; (C) high focal uptake in the left frontal lobe; (D) no other uptake areas in the body.

feration of spindle cells with nuclear pleomorphism displaying areas of necrosis, brain parenchymal invasion and a mitotic rate up to 20 mitosis/10 HPF. Immunohistochemical staining showed expression of EMA positivity and marked proliferative activity (Ki67 > 30\%). The tumor cells lacked expression for glial fibrillary acidic protein (GFAP), S-100 protein, Melan-A and CAM 5.2. These pathologic findings were consistent with the diagnosis of anaplastic meningioma and revealed a dedifferentiation of the tumor to a WHO grade III. The patient recovered well with minimal neurologic sequelae. Six months later in a follow-up MRI scan, the patient was diagnosed with a recurrence. After two cycles of chemotherapy with temozolomide (TMZ; Temodal; Merck; whitehouse Station, NJ) at a standard dose (200 $\mathrm{mg} / \mathrm{m}^{2}$ for 5 days, every 28 days), an indium-111-penteotride scintigraphy scan (Octreoscan; Covidien, Mansfield, MA) showed high, focal radionuclide tracer uptake in the left frontal lobe (Figure 1(C)) and an absence of other avid 111-In-penteotride uptake areas in the body (Figure 1(D)). Repeat surgery was evaluated as inappropriate and the patient underwent Cyberknife hypofractionated stereotactic radiosurgery (SRS). This treatment was followed by a therapy with antiangiogenic antibody bevacizumab $(10 \mathrm{mg} / \mathrm{kg}$ every 2 weeks) and octreotide acetate Long-Acting-Release (30mg every month) on the basis of Octreoscan results. One year later the SRS treatment and the start of target therapy, the patient complained mild pain on thorax right side with slight dyspnea. A computed tomography (CT) scan revealed a stable intracranial disease (Figure 2(A)), an extensive thickening of the right parietal pleura (Figure 2(B)), massive abdominal lymph nodes enlargement, and metastases in both hepatic lobes (Figure 2(C)). A fine needle aspiration biopsy (FNAB) of the pleural lesion resulted not diagnostic. An ultrasound guided core 

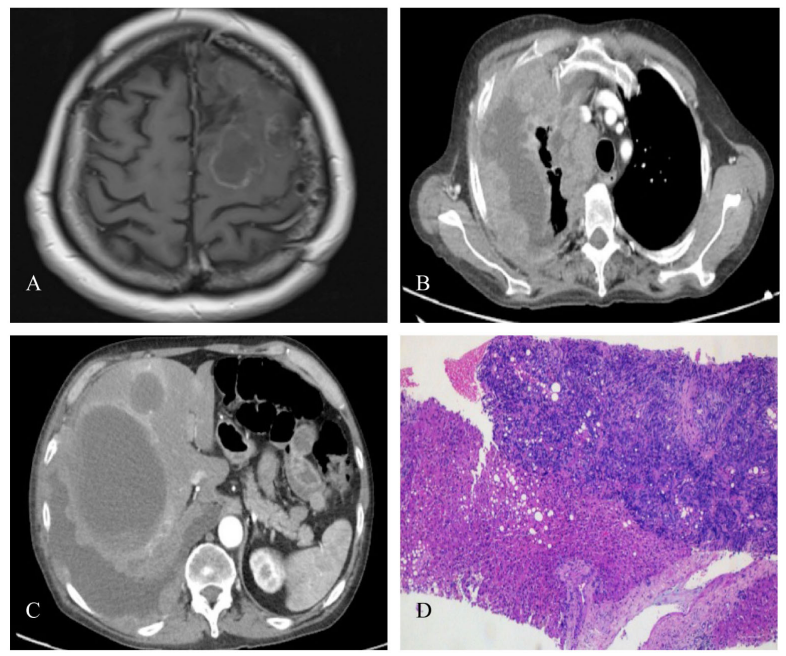

Figure 2. Computed Tomography (CT) and histological features of liver metastasis of anaplastic meningioma. (A) stable intracranial disease; (B) extensive right parietal pleural thickening; (C) metastases of both hepatic lobes; (D) spindle cell tumors with clear cells aspects.

biopsy of a hepatic mass revealed histologic features of spindle cell tumor with clear cells aspects (Figure 2(D)). Immunohistochemistry demonstrated EMA and Vimentin positivity and a Ki67 index of 30\%. The tumor cells lacked expression for GFAP, S-100 protein, Melan-A, anti-Melanoma CD56, DOG-1, TTF1, CD117, Synaptophisin, CK8/18, CKAE1-AE3, Progesterone receptor (PR) and CEA. These pathologic findings were consistent with the diagnosis of liver metastasis of AM-WHO-III ${ }^{\circ}$. In agreement with the patient and his relatives, treatment was halted and the patient was referred to supportive care. Notwithstanding appropriate care, the patient passed away 33 months after the first operation.

\section{Discussion}

Complete surgical excision is the treatment of choice for all resectable meningioma tumors. Postoperative radiotherapy is recommended to prevent local recurrence, especially in cases of partial resection or when histology suggests malignancy. The role of chemotherapy, as monotherapy or combination regimens, is limited to tumors that show no or minimal consistent response to radiotherapy. The management for recurrent meningioma includes second surgery, radiotherapy and stereotactic radiosurgery. Meningiomas are vascularized tumors and this represents the reason for the use of antiangiogenic therapy with bevacizumab [3]. 111-Indium labeled octreotide brain scintigraphy can be used to delineate disease extent and to select patients with inoperable recurrence refractory to radiotherapy who are likely to respond to somatostatin analogues therapy [4]. In fact, somatostatin receptors (SR) are present on most meningiomas. Only $0.1 \%-0.2 \%$ of all intracranial meningiomas metastasize outside the central nervous system with the lungs as preferred site [5]. Metastases other than pulmonary are rarely reported in the literature but they may occur in intra-abdominal organs, lymph nodes, bone, and muscle [6] [7]. Recurrent tumors with malignant histological 
features and a history of repeated surgical resections are associated with a higher risk of extracranial metastases [8]. In spite of this, a systemic workup for distant metastases is usually not included in the evaluation paradigm for patients with high-risk recurrent meningioma. The current case was a massive extracranial dissemination of an intracranial secondary AM-WHO-III ${ }^{\circ}$ and revealed as an unexpected diagnosis because of mild clinical signs without local intracranial progression. In our patient, the malignant progression according to the WHO grade, from atypical to anaplastic phenotype, was characterized by histological aspects of frank malignancy of the recurrent tumor and by a significant increase of the $\mathrm{Ki}-67$ index (from $10 \%$ in the original tumor to $30 \%$ in the recurrence) [9]. The increase of the $\mathrm{Ki}-67$ index showed a good correlation with the histological grade and the tendency of the tumor to recur and metastasize [10]. According to this report, a multivisceral metastatic spread may occur in absence of intracranial progression. Therefore it should be taken into account in the patient's follow-up that extracranial metastatic process in $\mathrm{AM}-\mathrm{WHO}-\mathrm{III}^{\circ}$ is not necessarily linked to intracranial relapse.

\section{Conclusion}

The histological grade and the Ki-67 index are the most predictive factors for both recurrence and extracranial metastases in secondary AM-WHO-III ${ }^{\circ}$. These tumors are difficult to manage. Chemotherapy, radiotherapy and surgery have all been described and remain under study although none of these treatments has managed to achieve more than a partial response [11]. At recurrence, there is no consensus on treatment options. In our case, two cycles of short-term treatment with the cytotoxic agent temozolomide were not long enough to show effectiveness while SRS was effective to control local tumor. Conversely, target therapy with bevacizumab and octreotide acetate LAR showed disappointing results in the metastatic spread control.

\section{Conflict of Interest}

All authors have no financial or others conflict to declare.

\section{Author's Contributions}

All authors contributed equally to this work, and all authors read and approved the final manuscript.

\section{Statement of Ethics}

Written informed consent was obtained from the patient's relative (son) for publication of this case report and any accompanying images.

\section{References}

[1] Claus, E.B., Bondy, M.L., Schildkraut, J.M., Wiemels, J.L., Wrensch, M. and Black, P.M. (2005) Epidemiology of Intracranial Meningioma. Neurosurgery, 57, 1088-1095. https:/doi.org/10.1227/01.NEU.0000188281.91351.B9 
[2] Juratli, T. Geiger, K. and Lautenschlaeger, T. (2014) Primary and Secondary Malignant Meningiomas: A Clinical and Histological Comparison. Journal of Clinical Oncology, 32, 5s.

[3] Sumrall, A.L. and Turner, S. (2012) Bevacizumab Therapy for Adults with Recurrent/Progressive Meningioma: A Retrospective Series. Journal of Neuro-Oncology, 109, 63-70.

https:/doi.org/10.1007/s11060-012-0861-0

[4] Fabi, A., Nuzzo, C., Vidiri, A., Ciccarese, M., Felici, A., Cattani, F. and Cognetti, F. (2006) Bone and Lung Metastases from Intracranial Meningioma. Anticancer Research, 26, 38353837.

[5] Chamberlain, M.C., Glantz, M.J. and Fadul, C.E. (2007) Recurrent Meningioma: Salvage Therapy with Long-Acting Somatostatin Analogue. Neurology, 69, 969-973.

https:/doi.org/10.1212/01.wnl.0000271382.62776.b7

[6] Kanthan, R. and Senger. J.L. (2013) Distant Metastases from Meningiomas: A Myth or Reality? Annals of Clinical Pathology, 1, 1001.

[7] Estanislau, E.S., Carvalho, G.T., Reis, B.L., et al. (2009) Malignant Meningioma with Extracranial Metastases. Arquivos de Neuro-Psiquiatria, 67, 730-732. https:/doi.org/10.1590/S0004-282X2009000400036

[8] Cachia, D., Alderson, L., Smith, T. and Yunus, S. (2012) Widely Metastatic Meningioma. Archives of Neurology, 69, 1372-1373. https:/doi.org/10.1001/archneurol.2012.1

[9] Bollag, R.J., Vender, J.R. and Sharma, S. (2010) Anaplastic Meningioma: Progression from Atypical and Chordoid Morphotype with Morphological Spectral Variation at Recurrence. Neuropathology, 30, 279-287. https:/doi.org/10.1111/j.1440-1789.2009.01060.x

[10] Torp, S.H., Lindboe, C.F., Grønberg, B.H., Lydersen, S. and Sundstrøm, S. (2005) Prognostic Significance of ki-67/mib-1 Proliferation Index in Meningiomas. Clinical Neuropathol$o g y, 24,170-174$.

[11] Wen, P.Y., Quant, E., Drappats, J., Beroukhim, R. and Norden, A.D. (2010) Medical Therapies for Meningiomas. Journal of Neuro-Oncology, 99, 365-378.

https:/doi.org/10.1007/s11060-010-0349-8

\section{Submit or recommend next manuscript to OALib Journal and we will provide best} service for you:

- Publication frequency: Monthly

- 9 subject areas of science, technology and medicine

- Fair and rigorous peer-review system

- Fast publication process

- Article promotion in various social networking sites (LinkedIn, Facebook, Twitter, etc.)

- Maximum dissemination of your research work

Submit Your Paper Online: Click Here to Submit

Or Contact service@oalib.com 